\title{
Clinical Perspectives of Theranostics
}

\author{
Shozo Okamoto ${ }^{1,2}$, Tohru Shiga ${ }^{3}$, and Nagara Tamaki ${ }^{4, *}$ \\ 1 Department of Radiology, Obihiro-Kosei General Hospital, Japan \\ 2 Department of Diagnostic Radiology, Hokkaido University Graduate School of Medicine, Japan \\ 3 Advanced Clinical Research center, Fukushima Global Medical Science Center, Japan \\ 4 Advanced Clinical Research center, Fukushima Global Medical Science Center, Japan \\ * Correspondence: natamaki@koto.kpu-m.ac.jp
}

\begin{abstract}
Theranostics covers combination of diagnostic and therapeutic techniques for various cancers throughout body using suitable drug combination. This review covers well-known treatment of thyroid cancer and pheochromocytoma with I-131 compounds and also new radiopharmaceuticals for prostatic cancer and pancreatic cancer. Of particular, new trends toward patient outcome has been focused. A recent clinical study highlighted the ability of alpha-radiotherapy with high LET to overcome treatment resistance to beta--particle therapy. The theranostics will become an ever-increasing part of clinical nuclear medicine these days.
\end{abstract}

Keywords: PET, theranostics, radionuclide therapy, cancer, prognosis

\section{Introduction}

Theranostics is a re-emerging new medical term of combination of diagnostic and therapeutic techniques mainly for oncology areas using suitable drug combination [1]. Theranostics has long been applied to treat thyroid cancer and neuroblastomas using suitable radiolabeled compounds, I-131 iodine and I-131-iodine-meta-iodobenzylguanidine (MIBG). More recently, theranostics has been refocused with advent of new therapeutic antibodies and small molecules which can be transformed into theranostic agents through radioconjugating with radioactive isotope materials [2]. A number of PET compounds labeled with Ga-68 or F-18 have been used for imaging, while beta- or alfa-emitting sister compounds are applied for therapy [3-5].

The theranostics plays an important role for both detection of malignant lesions throughout the body using tumor affinity compounds, and also treating lesions with radiotherapy emitted from beta- or alfa-emission from the same targeted radiolabeled compounds. Table 1 summarizes various combination of diagnostic imaging and internal therapy with radiopharmaceuticals for the same target molecule. Thus, systematic imaging and radioisotope therapy is permitted with use of suitable radiolabeled compounds. There are a number of reports indicating not only the diagnostic values but also better prognostic values of oncology therapy as compared to the conventional systemic chemotherapy $[6,7]$.

Table 1. Combination of diagnostic imaging and internal radiation therapy with radiopharmaceuticals for the same targe disease.

\begin{tabular}{ccc}
\hline Target Disease & Diagnosis & Therapy \\
\hline Thyroid cancer & I-131 & I-131 \\
\hline Malignant pheochromocytoma, & I-123 MIBG & I-131 MIBG \\
Neuroblastoma & I-131 MIBG & \\
\hline \multirow{2}{*}{ Neuroendocrine tumor } & In-111 Pentetreotide & Y-90 DOTATATE, \\
& Ga-68 DOTATE & Y-90 DOTATOC \\
& Ga-68 DOTATOC & Lu-177 DOTATATE \\
\hline
\end{tabular}


Lu-177 DOTATOC

\begin{tabular}{ccc}
\hline Malignant lymphoma & $\begin{array}{c}\text { In-111 lbritumomab } \\
\text { Tiuxetan } \\
\text { I-131 rituximab }\end{array}$ & $\begin{array}{c}\text { Y-90 Ibritumomab } \\
\text { Tiuxetan } \\
\text { Lu-177 rituximab }\end{array}$ \\
\hline $\begin{array}{c}\text { Bone metastasis of prostatic } \\
\text { cancer }\end{array}$ & Tc-99m bone scan & Sm-153 EDTMP \\
Ra-223 \\
\hline Prostatic cancer & Ga-68 PSMA & Lu-177 PSMA \\
& F-18 PSMA & Ac-225 PSMA \\
\hline
\end{tabular}

\section{Prognostic Value of Theranostics for Thyroid Cancer}

Radioiodine therapy with I-131 iodine is a well-established treatment modality for adjuvant postsurgical ablation of thyroid remnant tissue and therapy of nonresectable local recurrences, lymph node, and distant metastases [8].

Differentiated thyroid cancer has strong affinity to I-131 and thus, shows good response to iodine treatment. Dedifferentiated thyroid cancer lesions, on the other hand, tend to lose radioiodine affinity, but instead show an increased glucose metabolism [9]. Both histological and imaging studies provide evidence that increased glucose uptake correlates with a higher grade of malignancy and a worse prognosis [10-13]. Accordingly, FDG PET is considered a valuable tool for the detection of radioiodine-negative metastases in the follow-up of patients with DTC [14-17] (Figure 1 and Figure 2). In addition, FDG-PET holds a promise for valuable tool for predicting long prognosis of thyroid cancer.

We have analyzed the predictive value of FDG-PET in comparison with radioiodine uptake in 141 high-risk patients with differentiated thyroid cancer with radioiodine therapy after total thyroidectomy [18]. Our results indicate that FDG PET was more predictive for long-term survival, whereas radioiodine uptake was more important for short-term response. Therefore, FDG PET after at thyroid remnant ablation should hold a prognostic value for management of high-risk patients with differentiated thyroid cancer [18].
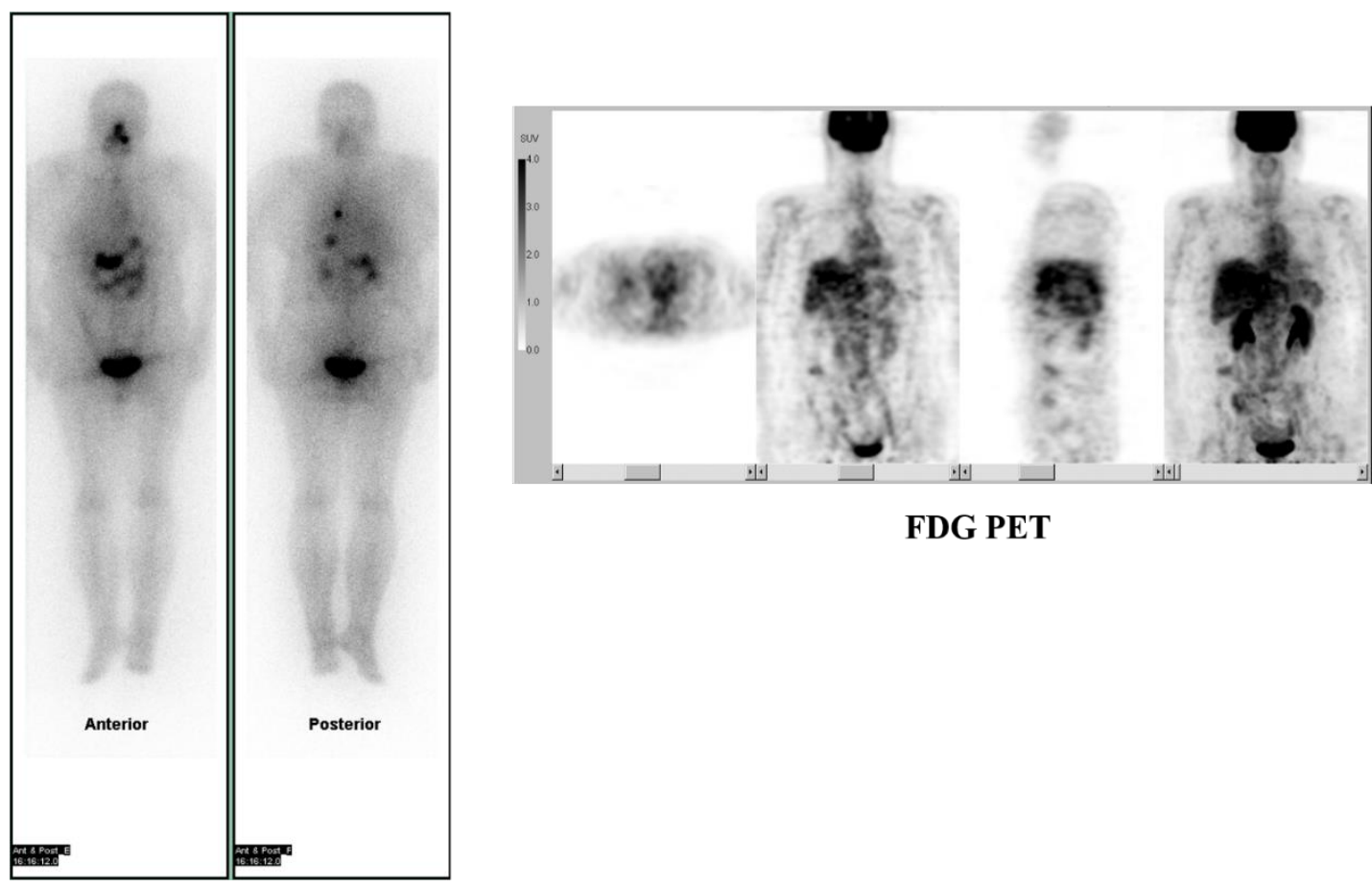

FDG PET 
Figure 1. A case with thyroid cancer showing positive I-131 and negative FDG uptake in the lung.

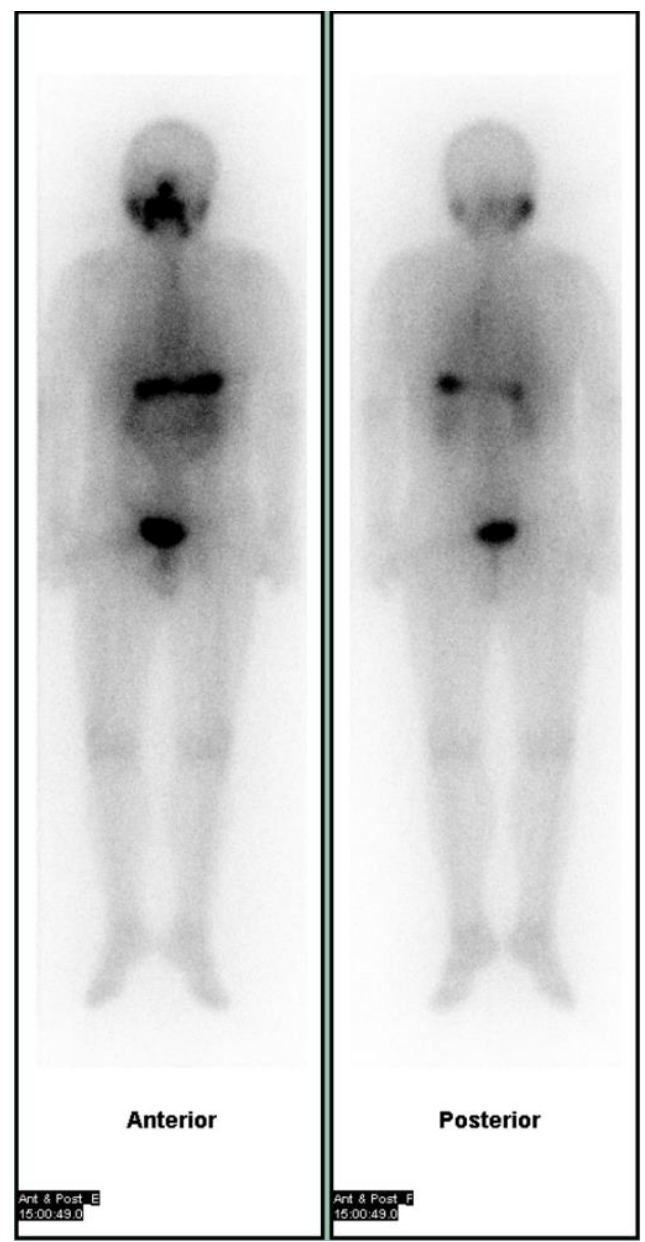

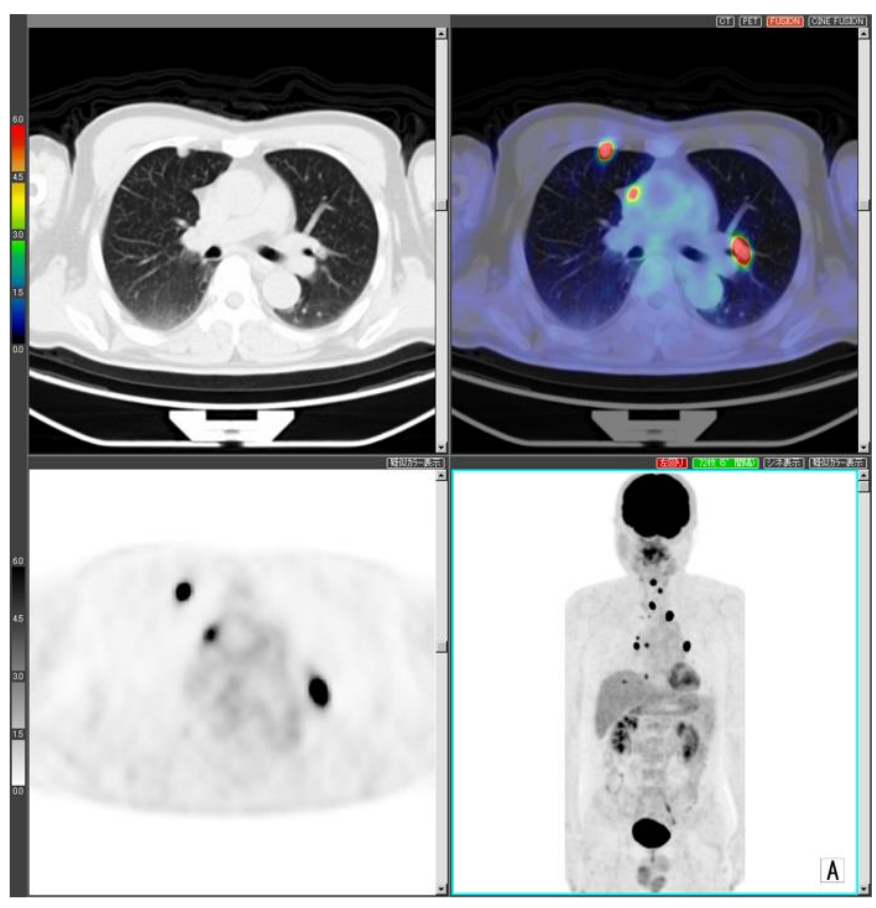

FDG PET-CT

Figure 2. A case with thyroid cancer showing negative I-131 and positive FDG uptake in the lung. 


\section{Prognostic Value of Theranostics for Neuroendocrine Tumor}

Pheochromocytoma and paraganglioma (PPGL) may develop metastatic lesions. The mean expected survival time with pheochromocytoma is 20.7 years and with paraganglioma is 9.8 years $[19,20]$. After surgery, systemic chemotherapy is commonly performed for treating remaining and metastatic lesions, however, the survival benefits are not clear because of the lack of prospective comparative studies [21-23].

Iodine-131-meta-iodobenzylguanidine (I-131 MIBG) is a substrate of the norepinephrine transporter system and the structure is similar to norepinephrine [24]. Norepinephrine works as a neurotransmitter in the central and autonomic nervous systems. The concentrations of norepinephrine in the nervous systems are regulated by the norepinephrine transporter. I-131 MIBG also accumulates; however, unlike norepinephrine, I-131 MIBG has little or no affinity for adrenergic receptors [25]. I-131 MIBG radiotherapy has been used to treat neuroendocrine tumors (NETs), including metastatic PPGL, medullary thyroid carcinoma and carcinoid tumors that have the uptake-1 mechanism [24,26,27].

I-131 MIBG radiotherapy has shown some survival benefits in metastatic NETs. European Association of Nuclear Medicine (EANM) clinical guidelines for I-131 MIBG radiotherapy suggest a repeated treatment protocol, although none currently exists [28]. The existing single-high-dose I-131 MIBG radiotherapy (444 MBq/kg) has been shown to have some benefits for patients with metastatic NETs. However, this protocol increases adverse effects and it requires alternative therapeutic approaches.

A previous study reported that FDG PET SUVmax was reduced after I-131 MIBG radiotherapy in patients considered to have responded, suggesting the value of FDG PET imaging for treatment evaluation in patients with PPGL after I-131 MIBG radiotherapy [29] (Figure 3).

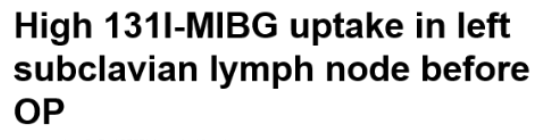
OP
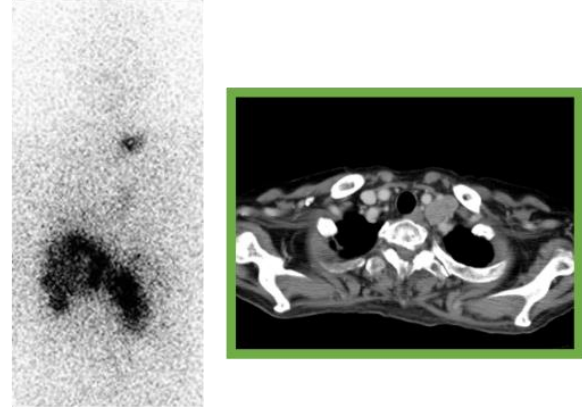

\section{Plasma norepinephrine $(\mathrm{pg} / \mathrm{mL})$} $40000 ; \mathbf{3 0 2 4 0}$

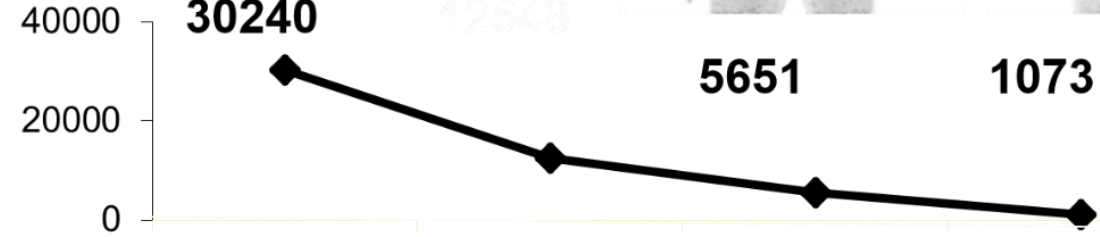

\section{and liver after OP}

\section{FDG-PET showing reduction of metastasis after MIBG therapy}

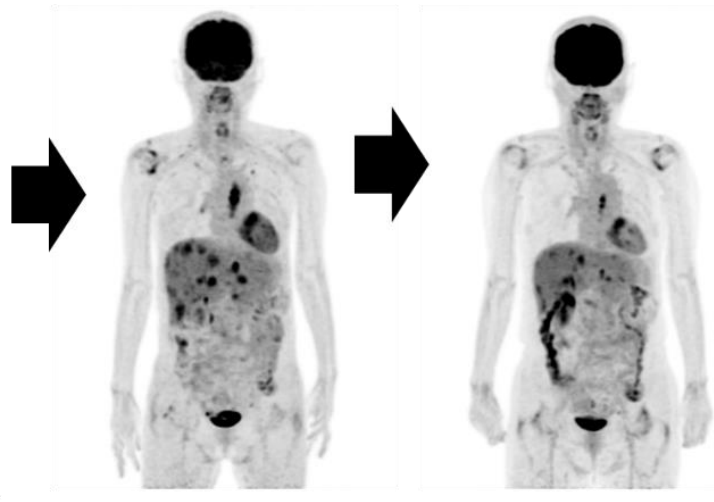

Figure 3. A case with pheochromocytoma.

Accordingly, we have evaluated the effects of repeated I-131 MIBG therapy on tumor size and tumor metabolic response in patients with metastatic NETs to find reduced tumor size and tumor metabolic activities according to lesion-based analysis in about onethird of patients and stabilized a majority with this therapy [30]. Thus, we concluded that this relatively short-term repeated I-131 MIBG treatment may have potential as one option in a therapeutic protocol for metastatic NETs [30]. 
Theranostics has recently been covered NETs with somatostatin receptor targeting small molecule tracers such as Ga-68 DOTATATE PET, In-111 Pentetreotide, and Peptide Receptor Radionuclide Therapy (PRRT) with Lu-177 DOTATATE therapy. The patients with positive uptake in Ga-68 DOTATATE PET or In-111 Pentetreotide are indication for Lu-177 DOTATATE treatment in European Medicines Agency (EMA) and Food and Drug Administration (FDA). Progression free survival (PFS) rate at 20 months was significantly increased from $11 \%$ of patients in the control group to $65 \%$ in the Lu-177 DOTA-TATE group $(p<0.0001)$ [31].

\section{Prognostic Value of Radioimmunotherapy of Non-Hodgkin Lymphoma}

Non-Hodgkin lymphoma is a common malignancy where I-131 compound has been used for theranostics. Initially 131-iodine-tositumomab was used for imaging and Y-90-ibritumomab-tiuxetan (Zevalin) was used for therapy. However, this initial theranostic trials have failed to become incorporated into mainstream oncologist practice. More recently, Lymphoma is effectively treated with 131-iodine-anti-CD20 radioimmunotherapy (RIT) in relapsed/refractory disease following traditional R-CHOP chemotherapy [32,33]. There was no significant toxicity, and also this single-shot theranostic study seems to be superior to all standard therapies. The recent substitution of Lu-177 rituximab, in place of 131-iodine radiolabelling will facilitate outpatient therapy by minimising radiation exposure of medical staffs, while retaining the ability to perform individual prospective dosimetry [34]. A recent clinical study using Lu-177 lilotomab satetraxan anti-CD37 monoclonal antibody RIT suggested that tumour radiation absorbed doses comparable with those achieved with 131-iodine-tositumomab anti-CD20 Mab were attained, at red marrow doses below the 2 Gy threshold for myelotoxicity [35].

\section{Prognostic Value of Theranostics for Bone Metastasis}

The skeleton is the most common metastatic site in patients with advanced cancer, particularly in prostatic cancer. Pain is a major healthcare problem in patients with bone metastases. Bone-seeking radionuclides that selectively accumulate in the bone are used to treat cancer-induced bone pain. The goals of these guidelines are to assist nuclear medicine practitioners in evaluating patients who might be candidates for radionuclide treatment of bone metastases using beta-emitting radionuclides such as strontium-89 and Samarium-153 EDTMP [36].

On the other hand, theranostic approaches for osseous metastasis have long been focused for alpha therapy using Ra-223 for various solid tumors, such as thyroid cancer and prostatic cancer. It is important that radionuclide therapy has not only a palliative effect but also a potential for prolong progression-free and overall survival [37-39]. This important randomized control study nicely showed 6 months longer survival by Ra-223 therapy for bone metastasis from castrate-resistant prostatic cancer [37]. Accordingly, Japanese government has approved insurance coverage for Ra-223 therapy for those with multiple bone metastasis from prostatic cancer [40].

\section{Alpha vs Beta-emitting Isotope Radiochemistry}

As previously described, theranostics has been applied by using suitable therapeutic radioisotopes, including alpha- and beta-emission ones over the last century. Beta-emitting radioisotopes have the longest particle pathlength $(\leq 12 \mathrm{~mm})$ and lowest linear energy transfer (LET) $(\sim 0.2 \mathrm{keV} / \mu \mathrm{m})$, supporting the effectiveness in medium to large tumors. Long beta-particle range is advantageous in evenly distributing radiation dose in heterogeneous tumors. But it can also result in the irradiation of healthy tissue surrounding the tumor site causing relatively large side effect. Alpha-particles have a moderate pathlength (50-100 $\mu \mathrm{m})$ and high LET $(80 \mathrm{keV} / \mu \mathrm{m})$ that render them especially suitable for small neoplasms or micro-metastases with such smaller side effects in surrounding normal organ. 
A recent clinical study highlighted the ability of alpha-radiotherapy to overcome treatment resistance to beta-particle therapy, prompting a paradigm shift in the approach toward radionuclide therapy [41,42].

\section{Future Perspectives of Theranostics}

More recently, a number of new radio-labeled pharmaceutical compounds have been investigated for promoting theranosticis. One of the most exciting new theranostic agent is prostate specific membrane antigen (PSMA) binding compound for prostatic cancer developed at Heidelberg University and many researches worldwide [43]. Prostatic cancer is the second most common cancer in men worldwide with an estimated about a million $1,276,000$ new patients. About $30 \%$ of men experience biochemical recurrence, often progress to castration resistant prostate cancer (CRPC), and 359,000 deaths in 2018 [44,45].

The prognosis of the patients with CRPC has been better because of new drugs was developed in the last decade. Ga-68 or F-18 labelled PSMA has been used for whole body PET imaging to identify metastatic lesions. In addition, Lu-177 or Ac-225 labeled PSMA therapy is expected additional effect by unique mechanism using radiopharmaceutical. Lu-177 or Ac-225 PSMA therapies are main strategies on theranostics at present. PSMA PET detects recurrent and metastatic tumors more accurately than conventional imaging diagnostic tools such as computed tomography (CT), bone scintigraphy (BS), or F-18 Fluciclovine PET [46-48]. In the study of our group at Technical University of Munich, the accuracy of Ga-68 PSMA PET was 99.8\% which was significantly much higher than BS $(84.3 \%)$ and BS + Single Photon Emission Computed Tomography (SPECT). Another international trial of Ga-68 PSMA-PET in biochemical recurrence, 75\% of these patients had positive lesion detected, and both the positive predictive value and sensitivity were $92 \%$ [49]. FDA approved Ga-68 PSMA-PET for patients with suspected prostate cancer metastasis and with suspected prostate cancer recurrence based on elevated serum prostatespecific antigen (PSA) levels in December 2020. Our group also reported that similar uptake is shown between pretherapeutic Ga-68 PSMA-PET and Lu-177 PSMA therapy, therefore, Ga-68 PSMA PET avid lesions are expected the effect of Lu-177 PSMA therapy (Figure 4) [50]. Up to 4 cycles treatments with 6 weeks interval are standard course of Lu177 PSMA therapy considering for kidney absorbed dose at a risk organ [50].

A

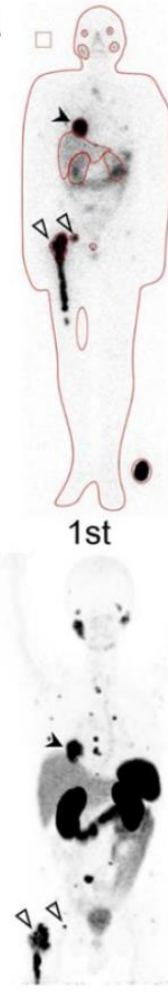

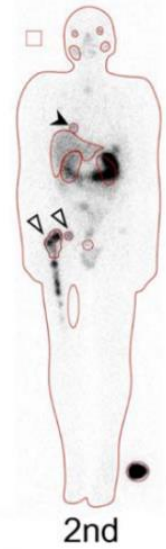

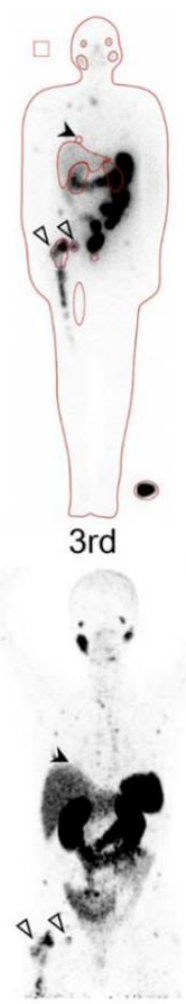
Figure 4. A patient with multiple metastases in lung (Black arrow) and bone (White arrows). A: posttherapeutic Lu-177 PSMA scintigraphy. B. Pretherapeutic Ga -68 PSMA PET (Okamoto et al. J Nucl Med 2017; 58: 445-450) [42].

Remarkable therapeutic effect of Lu-177 PSMA therapy for CRPC has been described in several reports [51-55]. PSA decline from baseline was seen in $68 \%$ of patients [47], greater than or equal to $50 \%$ seen in $44-64 \%$ of patients [51-55], At least $80 \%$ decline was seen in $44 \%$ of patients, and $16 \%$ achieved an at least $98 \%$ PSA decline (51). Regarding toxicity, no treatment-related deaths, and grade 2 or less xerostomia was reported in 8$66 \%$ of patients. Grade $1-2$ nausea was seen in $6-48 \%$, respectively. Grade $3-4$ toxicity was primarily hematologic, including leukopenia (3-32\%), thrombocytopenia (5-10\%), and anemia $(10 \%)$. Grade $1-2$ renal injury occurred in $10 \%$ of patients [51,52]. Ac-225 PSMA therapy is expected excellent therapeutic effect even for PC which resistant to Lu-177 PSMA therapy [56]. PSA decline of more than 50\% from baseline was observed in 21-63\% of the patients [57-59]. This rate is similar as the biochemical response rates of Lu-177 PSMA therapy, however, note that many patients who underwent Ac-225 PSMA therapy were refractory to previous Lu-177 PSMA therapy.

Another exciting theranostic trial is for applying for cancer with poor prognosis. Most of theranostic radiopharmaceuticals have so far been applied for cancer with relatively good prognosis. On the other hand, pancreatic adenocarcinoma has an extremely poor prognosis. Five years survival rate of metastatic pancreatic adenocarcinoma is less than 5\% [60]. At the time of diagnosis, most patients are ineligible for surgery due to metastatic spread pr local invasion. For metastatic disease cytotoxic chemotherapy is the only treatment choice in most occasion [61]. Neurotensin receptor 1 (is highly expressed in ductal pancreatic adenocarcinoma as well as hepatic metastasis but not in normal pancreatic tissue or chronic pancreatitis [61,62]. 3BP-227 is a DOTA-conjugated neurotensin receptor 1 antagonist [61] and Lu-177 labeled 3BP-227 significantly inhibited tumor growth [62]. The first clinical trial of Lu-177 labeled 3BP-227 indicated feasibility of treatment of ductal pancreatic adenocarcinoma [63]. More clinical experiences are warranted to see whether this new agent may prolong survival of this disease.

In external-radiation therapy, the radiation treatment plan is designed to focus the dose on the malignant tumor and to minimize damage to the surrounding tissues. On the other hand, in conventional RI therapy, doses are administered empirically, and there are no guidelines for dosimetry and no personalization. In RI-theranostics, dosimetry can be measured in the following two ways: one is to estimate the dose from the kinetic analysis of companion diagnostics, and the other is to measure the dose of the first treatment. AHASA (as high dose as safety administrated) approach is proposed for RI therapy [64]. Evidence on the relationship between dose and treatment or side effect is now gradually accumulating [65-68]. A study of theranostics for neuroendocrine tumors reported that dose escalation based on organ risk improved outcome [68]. In this study, they? conducted a prospective observational study of 200 patients with advanced metastatic NETs. Patients received additional cycles of treatment until the cumulative renal dose reached $23 \mathrm{~Gy}$ or was discontinued due to toxicity. Overall survival was significantly longer when treatment was given until the cumulative renal dose reached $23 \mathrm{~Gy}$. Once the evidence is established, a personalized, dosimetry-based treatment planning will become the mainstream for the purpose of more effective treatment and avoidance of serious side effects.

\section{Conclusion}

This review has outlined old as well as recently developed nuclear theranostics which has been entered the clinic as well as evolving imaging and therapy. Of particular, new trends toward patient outcome has been focused. The theranostics will become an ever-increasing part of clinical nuclear medicine these days.

Funding: none

Conflicts of Interest: The authors declare no conflict of interest 


\section{References}

1. Gilban I. THERANOSTICS an emerging tool in drug discovery and commercialisation. Drug Discover World 2002;Fall 1:1723.

2. DeNardo, G.L.; DeNardo, S.J. Concepts, Consequences, and Implications of Theranosis. Semin. Nucl. Med. 2012, 42, 147-150, doi:10.1053/j.semnuclmed.2011.12.003.

3. Sharma, H.; Mishra, P.K.; Talegaonkar, S.; Vaidya, B. Metal nanoparticles: a theranostic nanotool against cancer. Drug Discov. Today 2015, 20, 1143-1151, doi:10.1016/j.drudis.2015.05.009.

4. Feng, G.; Liu, B. Aggregation-Induced Emission (AIE) Dots: Emerging Theranostic Nanolights. Accounts Chem. Res. 2018, 51, 1404-1414, doi:10.1021/acs.accounts.8b00060.

5. Zhou, W.; Ding, J.; Liu, J. Theranostic DNAzymes. Theranostics 2017, 7, 1010-1025, doi:10.7150/thno.17736.

6. Siegel, R.L.; Miller, K.D.; Jemal, A. Cancer statistics, 2019. CA Cancer J. Clin. 2019, 69, 7-34, doi:10.3322/caac.21551.

7. Bray, F.; Ferlay, J.; Soerjomataram, I.; Siegel, R.L.; Torre, L.A.; Jemal, A. Global cancer statistics 2018: GLOBOCAN estimates of incidence and mortality worldwide for 36 cancers in 185 countries. CA Cancer J. Clin. 2018, 68, 394-424.

8. Luster, M.; Clarke, S.E.; Dietlein, M.; Lassmann, M.; Lind, P.; Oyen, W.J.G.; Tennvall, J.; Bombardieri, E. Guidelines for radioiodine therapy of differentiated thyroid cancer. Eur. J. Nucl. Med. Mol. Imaging 2008, 35, 1941-1959, doi:10.1007/s00259008-0883-1.

9. Feine, U.; Lietzenmayer, R.; Hanke, J.P.; Wöhrle, H.; Müller-Schauenburg, W. [18FDG whole-body PET in differentiated thyroid carcinoma. Flipflop in uptake patterns of 18FDG and 131I]. Nukl. 1995, 34, 127-134.

10. Schoenberger, J.; Rüschoff, J.; Grimm, D.; Marienhagen, J.; Rümmele, P.; Meyringer, R.; Kossmehl, P.; Hofstaedter, F.; Eilles, C. Glucose Transporter 1 Gene Expression is Related to Thyroid Neoplasms with an Unfavorable Prognosis: An Immunohistochemical Study. Thyroid. 2002, 12, 747-754, doi:10.1089/105072502760339307.

11. Robbins, R.J.; Wan, Q.; Grewal, R.K.; Reibke, R.; Gonen, M.; Strauss, H.W.; Tuttle, R.M.; Drucker, W.; Larson, S.M. RealTime Prognosis for Metastatic Thyroid Carcinoma Based on 2-[18F]Fluoro-2-Deoxy-d-Glucose-Positron Emission Tomography Scanning. J. Clin. Endocrinol. Metab. 2006, 91, 498-505, doi:10.1210/jc.2005-1534.

12. Deandreis, D.; Al Ghuzlan, A.; Leboulleux, S.; Lacroix, L.; Garsi, J.P.; Talbot, M.; Lumbroso, J.; Baudin, E.; Caillou, B.; Bidart, J.M.; et al. Do histological, immunohistochemical, and metabolic (radioiodine and fluorodeoxyglucose uptakes) patterns of metastatic thyroid cancer correlate with patient outcome? Endocrine-Related Cancer 2010, 18, 159-169, doi:10.1677/erc-100233.

13. Yoshio, K.; Sato, S.; Okumura, Y.; Katsui, K.; Takemoto, M.; Suzuki, E.; Katayama, N.; Kaji, M.; Kanazawa, S. The Local Efficacy of I-131 for F-18 FDG PET Positive Lesions in Patients With Recurrent or Metastatic Thyroid Carcinomas. Clin. Nucl. Med. 2011, 36, 113-117, doi:10.1097/rlu.0b013e318203bb6c.

14. Stokkel, M.P.M.; Duchateau, C.S.J.; Dragoiescu, C. The value of FDG-PET in the follow-up of differentiated thyroid cancer: a review of the literature. Q. J. Nucl. Med. Mol. Imaging 2006, 50, 78-87.

15. Reske, S.N.; Kotzerke, J. FDG-PET for clinical use. Eur. J. Nucl. Med. Mol. Imaging 2001, 28, 1707-1723, doi:10.1007/s002590100626.

16. Nanni, C.; Rubello, D.; Fanti, S.; Farsad, M.; Ambrosini, V.; Rampin, L.; Banti, E.; Carpi, A.; Muzzio, P.; Franchi, R. Role of 18F-FDG-PET and PET/CT imaging in thyroid cancer. Biomed. Pharmacother. 2006, 60, 409-413, doi:10.1016/j.biopha.2006.07.008.

17. Shiga, T.; Tsukamoto, E.; Nakada, K.; Morita, K.; Kato, T.; Mabuchi, M.; Yoshinaga, K.; Katoh, C.; Kuge, Y.; Tamaki, N. Comparison of (18)F-FDG, (131)I-Na, and (201)Tl in diagnosis of recurrent or metastatic thyroid carcinoma. J. Nucl. Med. 2001, 42, 414-419.

18. Gaertner, F.C.; Okamoto, S.; Shiga, T.; Ito, Y.M.; Uchiyama, Y.; Manabe, O.; Hattori, N.; Tamaki, N. FDG PET Performed at Thyroid Remnant Ablation Has a Higher Predictive Value for Long-Term Survival of High-Risk Patients With Well-Differentiated Thyroid Cancer Than Radioiodine Uptake. Clin. Nucl. Med. 2015, 40, 378-383, doi:10.1097/rlu.0000000000000699.

19. Neumann HPH, Young WF, Jr., Eng C. Pheochromocytoma and Paraganglioma. N Engl J Med. 2019;381, 552-565.

20. Ayala-Ramirez, M.; Feng, L.; Johnson, M.M.; Ejaz, S.; Habra, M.A.; Rich, T.; Busaidy, N.; Cote, G.J.; Perrier, N.D.; Phan, A.; et al. Clinical Risk Factors for Malignancy and Overall Survival in Patients with Pheochromocytomas and Sympathetic Paragangliomas: Primary Tumor Size and Primary Tumor Location as Prognostic Indicators. J. Clin. Endocrinol. Metab. 2011, 96, 717-725, doi:10.1210/jc.2010-1946.

21. Lenders, J.W.; Eisenhofer, G.; Mannelli, M.; Pacak, K. Phaeochromocytoma. Lancet 2005, 366, 665-675, doi:10.1016/s01406736(05)67139-5.

22. Jimenez, P.; Tatsui, C.; Jessop, A.; Thosani, S.; Jimenez, C. Treatment for Malignant Pheochromocytomas and Paragangliomas: 5 Years of Progress. Curr. Oncol. Rep. 2017, 19, 83, doi:10.1007/s11912-017-0643-0.

23. Björklund, P.; Pacak, K.; Crona, J. Precision medicine in pheochromocytoma and paraganglioma: current and future concepts. J. Intern. Med. 2016, 280, 559-573, doi:10.1111/joim.12507.

24. Taieb D, Jha A, Treglia G, Pacak K. Molecular imaging and radionuclide therapy of paraganglioma and pheochromocytoma. Endocr Relat Cancer. 2019.

25. Jimenez, C.; Erwin, W.D.; Chasen, B. Targeted Radionuclide Therapy for Patients with Metastatic Pheochromocytoma and Paraganglioma: From Low-Specific-Activity to High-Specific-Activity Iodine-131 Metaiodobenzylguanidine. Cancers 2019, 11, 1018, doi:10.3390/cancers11071018. 
26. Jackson, M.R.; Falzone, N.; A Vallis, K. Advances in Anticancer Radiopharmaceuticals. Clin. Oncol. 2013, 25, 604-609, doi:10.1016/j.clon.2013.06.004.

27. Kinuya, S.; Yoshinaga, K.; Higuchi, T.; Jinguji, M.; Kawamoto, H.; Kurihara, H. [Draft guideline regarding appropriate use of 131I-MIBG radiotherapy for neuroendocrine tumors Drafting Committee for Guideline of Radiotherapy with 131I-MIBG, Committee for Nuclear Oncology and Immunology, The Japanese Society of Nuclear Medicine]. Kaku igaku. Jpn. J. Nucl. Med. 2015, 52, 543-552.

28. Giammarile F, Chiti A, Lassmann M, Brans B, Flux G. EANM procedure guidelines for 131I-meta-iodobenzylguanidine (131I-mIBG) therapy. Eur J. Nucl. Med Mol Imaging. 2008;35:1039-1047.

29. Tokue, A.; Higuchi, T.; Oriuchi, N.; Arisaka, Y.; Endo, K. Clinical significance of 2-[18F]fluoro-2-deoxy-d-glucose positron emission tomography for the assessment of 131I-metaiodobenzylguanidine therapy in malignant phaeochromocytoma. Eur. J. Nucl. Med. Mol. Imaging 2011, 38, 1869-1875, doi:10.1007/s00259-011-1872-3.

30. Yoshinaga, K.; Abe, T.; Okamoto, S.; Uchiyama, Y.; Manabe, O.; Ito, Y.M.; Tamura, N.; Ito, N.; Yoshioka, N.; Washino, K.; et al. Effects of Repeated 131I-meta-iodobenzylguanidine Radiotherapy on Tumor Size and Tumor Metabolic Activities in Patients with Metastatic Neuroendocrine Tumors. J. Nucl. Med. 2020, doi:10.2967/jnumed.120.250803.

31. Strosberg, J.; El-Haddad, G.; Wolin, E.; Hendifar, A.; Yao, J.; Chasen, B.; Mittra, E.; Kunz, P.L.; Kulke, M.H.; Jacene, H.; et al. Phase 3 Trial of 177Lu-Dotatate for Midgut Neuroendocrine Tumors. N. Engl. J. Med. 2017, 376, 125-135, doi:10.1056/nejmoa1607427.

32. Leahy MF, Turner JH. Radioimmunotherapy of relapsed indolent non-Hodgkin lymphoma with 131I-rituximab in routine clinical practice: 10-year single-institution experience of 142 consecutive patients. Blood 2011; 117: 45-52.

33. McQuillan AD, Macdonald WB, Turner JH. Phase II study of first-line (131)I- rituximab radioimmunotherapy in follicular non-Hodgkin lymphoma and prognostic (18)F-fluorodeoxyglucose positron emission tomography. Leuk Lymphoma 2015; 56: 1271-7.

34. Kesavan M, McQuillan AD, Macdonald WBG, Turner JH. First-line radioimmunotherapy of advanced follicular non-Hodgkin lymphoma with 131-I-rituximab; Ten year follow-up. Leukemia E Lymphoma 2017; 35 (S2): 366-7.

35. Forrer F, Oechslin-Oberholzer C, Campana B, Herrmann R, Maecke HR, Mueller-Brand J, et al. Radioimmunotherapy with 177Lu-DOTA-rituximab: final results of a phase I/II Study in 31 patients with relapsing follicular, mantle cell, and other indolent B-cell lymphomas. J Nucl Med 2013; 54: 1045-52.

36. Bauman G, Charette M, Reid R, Sathya J.. "Radiopharmaceuticals for the palliation of painful bone metastases - a systematic review". Radiotherapy and Oncology. 2005; 75 (3): 258.E1-258.E13.

37. Parker, C.; Nilsson, S.; Heinrich, D.; Helle, S.; O’Sullivan, J.; Fosså, S.; Chodacki, A.; Wiechno, P.; Logue, J.; Seke, M.; et al. Alpha Emitter Radium-223 and Survival in Metastatic Prostate Cancer. New Engl. J. Med. 2013, 369, 213-223, doi:10.1056/nejmoa1213755.

38. Du, Y.; Carrio, I.; De Vincentis, G.; Fanti, S.; Ilhan, H.; Mommsen, C.; Nitzsche, E.; Sundram, F.; Vogel, W.; Oyen, W.; et al. Practical recommendations for radium-223 treatment of metastatic castration-resistant prostate cancer. Eur. J. Nucl. Med. Mol. Imaging 2017, 44, 1671-1678, doi:10.1007/s00259-017-3756-7.

39. Maffioli, L.S.; Florimonte, L.; Costa, D.C.; Castanheira, J.C.; Grana, C.; Luster, M.; Bodei, L.; Chinol, M. New radiopharmaceutical agents for the treatment of castration-resistant prostate cancer. Q J Nucl Med Mol Imaging 2015, 59.

40. Hosono, M.; Ikebuchi, H.; Nakamura, Y.; Yanagida, S.; Kinuya, S. Introduction of the targeted alpha therapy (with Radium223) into clinical practice in Japan: learnings and implementation. Ann. Nucl. Med. 2019, 33, 211-221, doi:10.1007/s12149-0181317-1.

41. Kratochwil C, Bruchertseifer F, Giesel FL, et al. ${ }^{225}$ Ac-PSMA-617 for PSMA-targeted $\alpha$-radiation therapy of metastatic castration-resistant prostate cancer. J Nucl Med. 2016;57:1941-1944.

42. Poty S, Francescpni LC, McDevitt MR, Morris MJ, Lewis JS. $\alpha$-emisstiers for radiotherapy: from basic radiochemistry to clinical studies. Part 1. J Nucl Med 2018; 59: 878-884

43. Afshar-Oromieh, A.; Haberkorn, U.; Eder, M.; Eisenhut, M.; Zechmann, C.M. [68Ga]Gallium-labelled PSMA ligand as superior PET tracer for the diagnosis of prostate cancer: comparison with 18F-FECH. Eur. J. Nucl. Med. Mol. Imaging 2012, 39, 1085-1086, doi:10.1007/s00259-012-2069-0.

44. Culp, M.; Soerjomataram, I.; Efstathiou, J.A.; Bray, F.; Jemal, A. Recent Global Patterns in Prostate Cancer Incidence and Mortality Rates. Eur. Urol. 2020, 77, 38-52, doi:10.1016/j.eururo.2019.08.005.

45. Center, M.M.; Jemal, A.; Lortet-Tieulent, J.; Ward, E.; Ferlay, J.; Brawley, O.W.; Bray, F. International Variation in Prostate Cancer Incidence and Mortality Rates. Eur. Urol. 2012, 61, 1079-1092, doi:10.1016/j.eururo.2012.02.054.

46. Eiber M, Maurer T, Souvatzoglou M, et al. Evaluation of Hybrid ${ }^{68} \mathrm{Ga}-\mathrm{PSMA}$ Ligand PET/CT in 248 Patients with Biochemical Recurrence After Radical Prostatectomy. J. Nucl. Med. 2015;56, 668-74.

47. Pyka, T.; Okamoto, S.; Dahlbender, M.; Tauber, R.; Retz, M.; Heck, M.; Tamaki, N.; Schwaiger, M.; Maurer, T.; Eiber, M. Comparison of bone scintigraphy and 68Ga-PSMA PET for skeletal staging in prostate cancer. Eur. J. Nucl. Med. Mol. Imaging 2016, 43, 2114-2121, doi:10.1007/s00259-016-3435-0.

48. Calais, J.; Ceci, F.; Eiber, M.; A Hope, T.; Hofman, M.S.; Rischpler, C.; Bach-Gansmo, T.; Nanni, C.; Savir-Baruch, B.; Elashoff, D.; et al. 18F-fluciclovine PET-CT and 68Ga-PSMA-11 PET-CT in patients with early biochemical recurrence after prostatectomy: a prospective, single-centre, single-arm, comparative imaging trial. Lancet Oncol. 2019, 20, 1286-1294, doi:10.1016/s1470-2045(19)30415-2. 
49. Fendler WP,; Calais J,; Eiber M,; Flavell RR,; Mishoe A,; Feng FY,; Nguyen HG,; Reiter RE,; Rettig MB,; Okamoto S,; et al. Assessment of 68Ga-PSMA-11 PET Accuracy in Localizing Recurrent Prostate Cancer: A Prospective Single-Arm Clinical Trial. JAMA Oncol. 2019, 5, 856-863. doi:10.1001/jamaoncol.2019.0096.

50. Okamoto, S.; Thieme, A.; Allmann, J.; D'Alessandria, C.; Maurer, T.; Retz, M.; Tauber, R.; Heck, M.M.; Wester, H.-J.; Tamaki, N.; et al. Radiation Dosimetry for 177 Lu-PSMA I\&T in Metastatic Castration-Resistant Prostate Cancer: Absorbed Dose in Normal Organs and Tumor Lesions. J. Nucl. Med. 2016, 58, 445-450, doi:10.2967/jnumed.116.178483.

51. Violet, J.; Sandhu, S.; Iravani, A.; Ferdinandus, J.; Thang, S.-P.; Kong, G.; Kumar, A.R.; Akhurst, T.; A Pattison, D.; Beaulieu, A.; et al. Long-Term Follow-up and Outcomes of Retreatment in an Expanded 50-Patient Single-Center Phase II Prospective Trial of 177Lu-PSMA-617 Theranostics in Metastatic Castration-Resistant Prostate Cancer. J. Nucl. Med. 2019, 61, 857-865, doi:10.2967/jnumed.119.236414.

52. Rahbar, K.; Ahmadzadehfar, H.; Kratochwil, C.; Haberkorn, U.; Schäfers, M.; Essler, M.; Baum, R.P.; Kulkarni, H.R.; Schmidt, M.; Drzezga, A.; et al. German Multicenter Study Investigating 177 Lu-PSMA-617 Radioligand Therapy in Advanced Prostate Cancer Patients. J. Nucl. Med. Off. Publ. Soc. Nucl. Med. 2016, 58, 85-90, doi:10.2967/jnumed.116.183194.

53. Ahmadzadehfar, H.; Wegen, S.; Yordanova, A.; Fimmers, R.; Kürpig, S.; Eppard, E.; Wei, X.; Schlenkhoff, C.; Hauser, S.; Essler, M. Overall survival and response pattern of castration-resistant metastatic prostate cancer to multiple cycles of radioligand therapy using [177Lu]Lu-PSMA-617. Eur. J. Nucl. Med. Mol. Imaging 2017, 44, 1448-1454, doi:10.1007/s00259-0173716-2.

54. Bräuer, A.; Grubert, L.S.; Roll, W.; Schrader, A.J.; Schäfers, M.; Bögemann, M.; Rahbar, K. 177Lu-PSMA-617 radioligand therapy and outcome in patients with metastasized castration-resistant prostate cancer. Eur. J. Nucl. Med. Mol. Imaging 2017, 44, 1663-1670, doi:10.1007/s00259-017-3751-z.

55. Baum, R.P.; Kulkarni, H.R.; Schuchardt, C.; Singh, A.; Wirtz, M.; Wiessalla, S.; Schottelius, M.; Mueller, D.; Klette, I.; Wester, H.-J. 177Lu-Labeled Prostate-Specific Membrane Antigen Radioligand Therapy of Metastatic Castration-Resistant Prostate Cancer: Safety and Efficacy. J. Nucl. Med. 2016, 57, 1006-1013, doi:10.2967/jnumed.115.168443.

56. Kratochwil, C.; Bruchertseifer, F.; Giesel, F.L.; Weis, M.; A Verburg, F.; Mottaghy, F.; Kopka, K.; Apostolidis, C.; Haberkorn, U.; Morgenstern, A. 225Ac-PSMA-617 for PSMA-Targeted -Radiation Therapy of Metastatic Castration-Resistant Prostate Cancer. J. Nucl. Med. 2016, 57, 1941-1944, doi:10.2967/jnumed.116.178673.

57. Kratochwil, C.; Bruchertseifer, F.; Rathke, H.; Hohenfellner, M.; Giesel, F.L.; Haberkorn, U.; Morgenstern, A. Targeted $\alpha-$ Therapy of Metastatic Castration-Resistant Prostate Cancer with 225Ac-PSMA-617: Swimmer-Plot Analysis Suggests Efficacy Regarding Duration of Tumor Control. J. Nucl. Med. 2018, 59, 795-802, doi:10.2967/jnumed.117.203539.

58. Yadav, M.P.; Ballal, S.; Sahoo, R.K.; Tripathi, M.; Seth, A.; Bal, C. Efficacy and safety of 225Ac-PSMA-617 targeted alpha therapy in metastatic castration-resistant Prostate Cancer patients. Theranostics 2020, 10, 9364-9377, doi:10.7150/thno.48107.

59. Zacherl, M.J.; Gildehaus, F.J.; Mittlmeier, L.; Boening, G.; Gosewisch, A.; Wenter, V.; Schmidt-Hegemann, N.-S.; Belka, C.; Kretschmer, A.; Casuscelli, J.; et al. First clinical results for PSMA targeted alpha therapy using 225Ac-PSMA-I\&T in advanced mCRPC patients. J. Nucl. Med. 2020, doi:10.2967/jnumed.120.251017.

60. Ducreux, M.; Cuhna, A.S.; Caramella, C.; Hollebecque, A.; Burtin, P.C.; Goéré, D.; Seufferlein, T.; Haustermans, K.; Van Laethem, J.L.; Conroy, T.; et al. Cancer of the pancreas: ESMO Clinical Practice Guidelines for diagnosis, treatment and follow-up. Ann. Oncol. 2015, 26, v56-v68, doi:10.1093/annonc/mdv295.

61. Körner, M.; Waser, B.; Strobel, O.; Büchler, M.W.; Reubi, J.C. Neurotensin receptors in pancreatic ductal carcinomas. EJNMMI Res. 2015, 5, 1-7, doi:10.1186/s13550-015-0094-2.

62. Schulz J, Rohracker M, Stiebler M, et al. Proof of therapeutic efficacy of a novel 177Lu-labeled neurotensin receptor 1 antagonist in a colon carcinoma xenograft model. J. Nucl Med. 2017;58:936-941.

63. Baum, R.P.; Singh, A.; Schuchardt, C.; Kulkarni, H.R.; Klette, I.; Wiessalla, S.; Osterkamp, F.; Reineke, U.; Smerling, C. 177Lu3BP-227 for Neurotensin Receptor 1-Targeted Therapy of Metastatic Pancreatic Adenocarcinoma: First Clinical Results. J. Nucl. Med. 2017, 59, 809-814, doi:10.2967/jnumed.117.193847.

64. Chiesa C, Sjogreen Gleisner K, Flux G, Gear J, Walrand S, Bacher K, et al. The conflict between treatment optimization and registration of radiopharmaceuticals with fixed activity posology in oncological nuclear medicine therapy. European Journal of Nuclear Medicine and Molecular Imaging. 2017. pp. 1783-1786. doi:10.1007/s00259-017-3707-3

65. Svensson J, Hagmarker L, Magnander T, Wängberg B, Bernhardt P. Radiation exposure of the spleen during (177)Lu-DOTATATE treatment and its correlation with haematological toxicity and spleen volume. EJNMMI Phys. 2016;3: 15.

66. Bergsma H, Konijnenberg MW, Kam BLR, Teunissen JJM, Kooij PP, de Herder WW, et al. Subacute haematotoxicity after PRRT with (177)Lu-DOTA-octreotate: prognostic factors, incidence and course. Eur J Nucl Med Mol Imaging. 2016;43: 453463.

67. van den Hoven AF, Rosenbaum CENM, Elias SG, de Jong HWAM, Koopman M, Verkooijen HM, et al. Insights into the Dose-Response Relationship of Radioembolization with Resin 90Y-Microspheres: A Prospective Cohort Study in Patients with Colorectal Cancer Liver Metastases. J Nucl Med. 2016;57: 1014-1019.

68. Garske-Román U, Sandström M, Baron KF, Lundin L, Hellman P, Welin S, et al. Prospective observational study of 177 LuDOTA-octreotate therapy in 200 patients with advanced metastasized neuroendocrine tumours (NETs): feasibility and impact of a dosimetry-guided study protocol on outcome and toxicity. Eur J Nucl Med Mol Imaging. 2018;45: 970-988. 